This is the final peer-reviewed accepted manuscript of:

S. V. Suraci, D. Fabiani and C. Li

Post-irradiation effect analysis on XLPE-insulated LV cables used in nuclear power plants

in 2nd International Conference on Electrical Materials and Power Equipment (ICEMPE), Guangzhou, China, 2019, pp. 53-56

The final published version is available online at:

https://doi.org/10.1109/ICEMPE.2019.8727281

Rights / License:

The terms and conditions for the reuse of this version of the manuscript are specified in the publishing policy. For all terms of use and more information see the publisher's website.

This item was downloaded from IRIS Università di Bologna (https://cris.unibo.it/)

When citing, please refer to the published version. 


\title{
Post-irradiation effect analysis on XLPE-insulated LV cables used in nuclear power plants
}

\author{
Simone Vincenzo Suraci ${ }^{*}$, Davide Fabiani ${ }^{1}$, Chuanyang $\mathrm{Li}^{1}$ \\ Department of Electrical Engineering, University of Bologna \\ Viale del Risorgimento, 2 Bologna, BO, Italy \\ Corresponding author: simone.suraci@unibo.it
}

\begin{abstract}
This paper investigates aging of low voltage cables used in nuclear power plants by the means of the dielectric spectroscopy technique. Aging has been performed on XLPE cables through high temperature and different dose rates in order to evaluate the electrical response under different aging conditions. Test have been carried out immediately after aging and years after the radiation source has been turned off. Significant change in dielectric response have been observed due to postirradiation effects, suggesting that degradation continues even after the aging source has been removed.
\end{abstract}

Keywords - XLPE, post-irradiation effect, permittivity, condition monitoring, electrical insulation, nuclear power plant

\section{INTRODUCTION}

Low-voltage electrical cables are extensively used throughout nuclear power plants (NPPs) for instrumentation and control of equipment, power transmission and communication of signals and data. These cables can be exposed to a extensive range of severe environmental conditions and stresses, like e.g., temperature, radiation, mechanical stresses and humidity, mainly inside the containment area.

Most of the NPP commissioned in '70s and '80s are now reaching end-of-life but, since decommissioning costs are very high, NPP regulators are trying to extend NPPs life up to other 60 (possibly even 80) years [1]. Since electric cables are one of the long-life equipment that have not considered for replacement during the design life of a NPP, usually 40 years, evaluating degradation condition and predicting residual life are very critical issues [1-3].

Cable insulation and jackets are made of polymer-based compounds, whose degradation state is traditionally assessed through destructive methods, like tensile testing or thermal analysis [2, 3]. Non-destructive techniques based e.g. on electrical quantities are not commonly used in NPP to evaluate cable insulation condition. This was the aim of the European project FP7 ADVANCE (Aging Diagnostics and Prognostics of low voltage I\&C cables). The project, finished in 2014, was focused on the study and the analysis of the results of accelerated aging tests performed on a representative selection of cables already installed in European NPPs in order to evaluate the ability of electrical Condition Monitoring (CM) techniques to detect local and global cable aging. The results were compared and correlated to those obtained with more conventional CM techniques for validation. Electrical permittivity, for instance, was found to be effectively associated with oxidation induced by aging $[3,4]$. Unfortunately, Diffusion Limited Oxidation (DLO) sometimes complicates the situation, causing inhomogeneous degradation of electrical insulation, which leads to unexpected behavior of electrical and physical properties of the insulating material with aging time $[5,6]$. Moreover, post-irradiation effects have been also observed which may change material properties several months or even years after irradiation, due to slow diffusion and reaction rate of some chemical radicals created by aging. Both phenomena must be taken into account to develop effective $\mathrm{CM}$ techniques for electrical insulation under such stress conditions [5, 6, 7].

In this paper, crosslinked polyethylene (XLPE) cable samples aged through high temperature and two different radiation levels have been analyzed through the dielectric spectroscopy technique in order to evaluate the change in electrical response of the insulation with aging.

Moreover, the same cables have been tested again after five years of storage in an uncontrolled environment in order to evaluate post-irradiation effects and long-term electrical response of cables subjected to different aging conditions.

\section{EXPERIMENTAL SETUP}

\section{A. Specimens}

I\&C Alcatel RG59B cable specimens with XLPE insulation for nuclear power plants are studied in this paper. Commercial low voltage (LV) cables are made of three components: the inner conductor (copper), the primary insulation (XLPE) and the outer sheath. In order to test the primary insulation, this latter has been removed from the specimens.

Sample cables have been aged through two different radiation doses and temperature for different time periods and then analyzed at delivery time and after five years during which they were stored in uncontrolled air conditions.

Cables analyzed in this paper are characterized by:

- Conductor: copper, $\varnothing 0.6 \mathrm{~mm}$

- Electrical insulation: XLPE, thickness $1.6 \mathrm{~mm}$

- Dose rate: $0.42 \mathrm{kGy} / \mathrm{h}$ and $1.06 \mathrm{kGy} / \mathrm{h}$

- Temperature: $85^{\circ} \mathrm{C}$ 
Nevertheless, forcing high dose rates, chemical reactions are limited by the time it takes for oxygen to diffuse into the material; that is why it may occur that aging treatment affects particularly the surface layer of specimens. This phenomenon, mentioned in the previous section, is known in literature as DLO and affected the specimens investigated in this paper due to the large thickness of cable insulation.

\section{B. Accelerated aging}

The accelerated aging procedure was carried out at the ROZA facility (UJV Rež, Czech Republic). through a ${ }^{60} \mathrm{Co} \gamma$-ray source with temperature set at $85^{\circ} \mathrm{C}$. The maximum aging time was $1000 \mathrm{~h}$ and specimens were removed for analysis every 200h.

\section{Measurements}

Dielectric response of specimens was analyzed in the range of $10^{-2}-10^{6} \mathrm{~Hz}$ through a NovoControl Alpha Dielectric Analyzer V2.2 with applied voltage of $3 \mathrm{~V}_{\text {rms. }}$. Instrumentation setup applies input voltage to the central metal conductor, measuring the output signal from a metallic shield placed on the external part of the insulation.

\section{RESULTS AND DISCUSSION}

Real and imaginary parts of permittivity measured at delivery time on cables aged at $85^{\circ} \mathrm{C}$ and with dose rate of $0.42 \mathrm{kGy} / \mathrm{h}$ and $1.06 \mathrm{kGy} / \mathrm{h}$ are reported in Figs. 1 and 2, respectively. It can be observed that in the low dose rate aging conditions, the real part of permittivity underlines a noticeable raise with the aging time in the high and middle frequency range (Fig. 1.a).

Referring to the high dose rate aging (Fig. 2.a), diffusion limited oxidation causes a very small raising of $\varepsilon$ ' with aging. Both these behaviors can be explained through the diffusion of the oxygen inside the polymer. The presence of high dose rates, in fact, prevents the diffusion of oxygen molecules in the inner part of specimens, so that oxygen groups can only be localized in the outer layers of the irradiated polymers, leading to the aforementioned DLO.

In the low frequency range $\varepsilon$ ' increase linearly with the lowering of frequency in both the aging conditions, probably due to electrode polarization effects as reported in literature [8]. This phenomenon can be explained through the presence of dissolved free ions inside the polymer blend which tend to move towards the electrode/sample interface, leading to the development of ionic double layers. Electrode polarization (EP) depends microscopically upon the electrode surface structure and area, as well as upon the surface chemistry (reactive surface groups or atoms). Higher dose rates (Fig. 1.a) brings to the shift to lower frequencies of the EP with respect to the lower DR.

The imaginary part of permittivity (Fig. 1.b and 2.b) shows the same behavior discussed about the real part of $\varepsilon$. Dielectric losses increase more widely with aging time for the low dose rate because the slow migration of oxygen in the polymer blend prevent the formation of DLO and the polymer is subject to chemical degradation not only on the surface but also in the bulk. In fact, higher dose rates (Fig. 2.b) cause a noticeable increase only during the first $400 \mathrm{~h}$ of aging, longer aging periods bring to negligible raise of $\varepsilon$ "' with aging time due to the DLO.
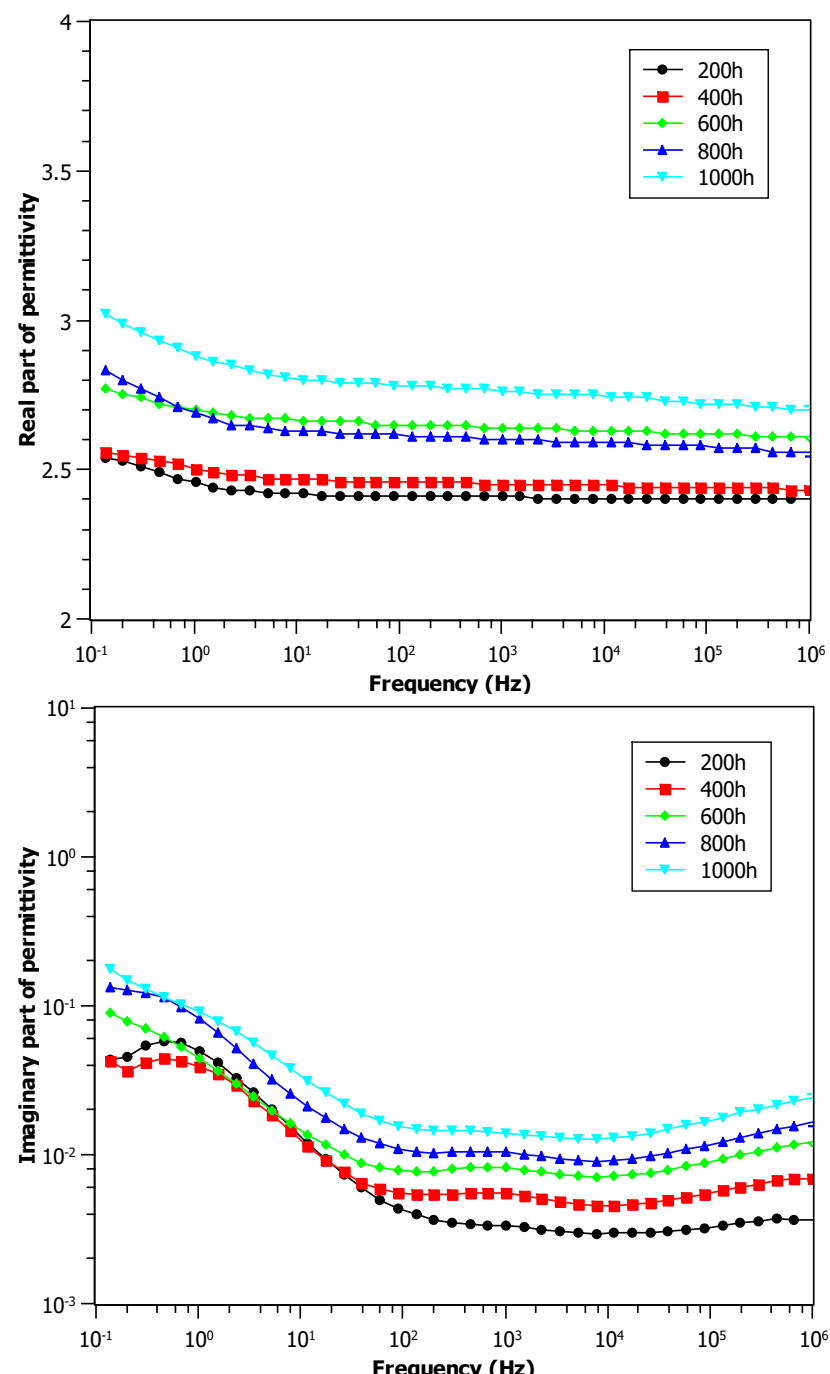

Fig. 1. Dielectric spectra as a fuction of frequency of real (a) and imaginary (b) part of permittivity. Dose rate $0.42 \mathrm{kGy} / \mathrm{h}$ and temperature $85{ }^{\circ} \mathrm{C}$. Tests made at delivery time.

The interfacial polarization can be evaluated through the analysis of the dielectric spectrum in the very low-frequency range where the two spectra (Figg. 1.b, 2.b) have quite different trend. As known $[6,7,9]$, irradiation causes the formation of radicals inside the polymer blend, obviously different dose rates create different kind and/or amount of radicals which can arrange differently inside the amorphous phase of the polymer. This causes the formation of different macromolecular structures which correspond to different interface arrangement and, consequently, different interfacial polarization. In particular, higher dose rates (Fig. 2.b) cause wider interfacial polarization peak with respect to the lower irradiation rate (Fig. 1.b) in which the peak is stiffer and slightly shifted towards lower frequencies. 
Cable specimens have been tested again after five years of storage in uncontrolled environment (Fig. 3 and 4) in order to analyze the possible post-irradiation effect which can happen inside the polymer insulation $[6,7]$.
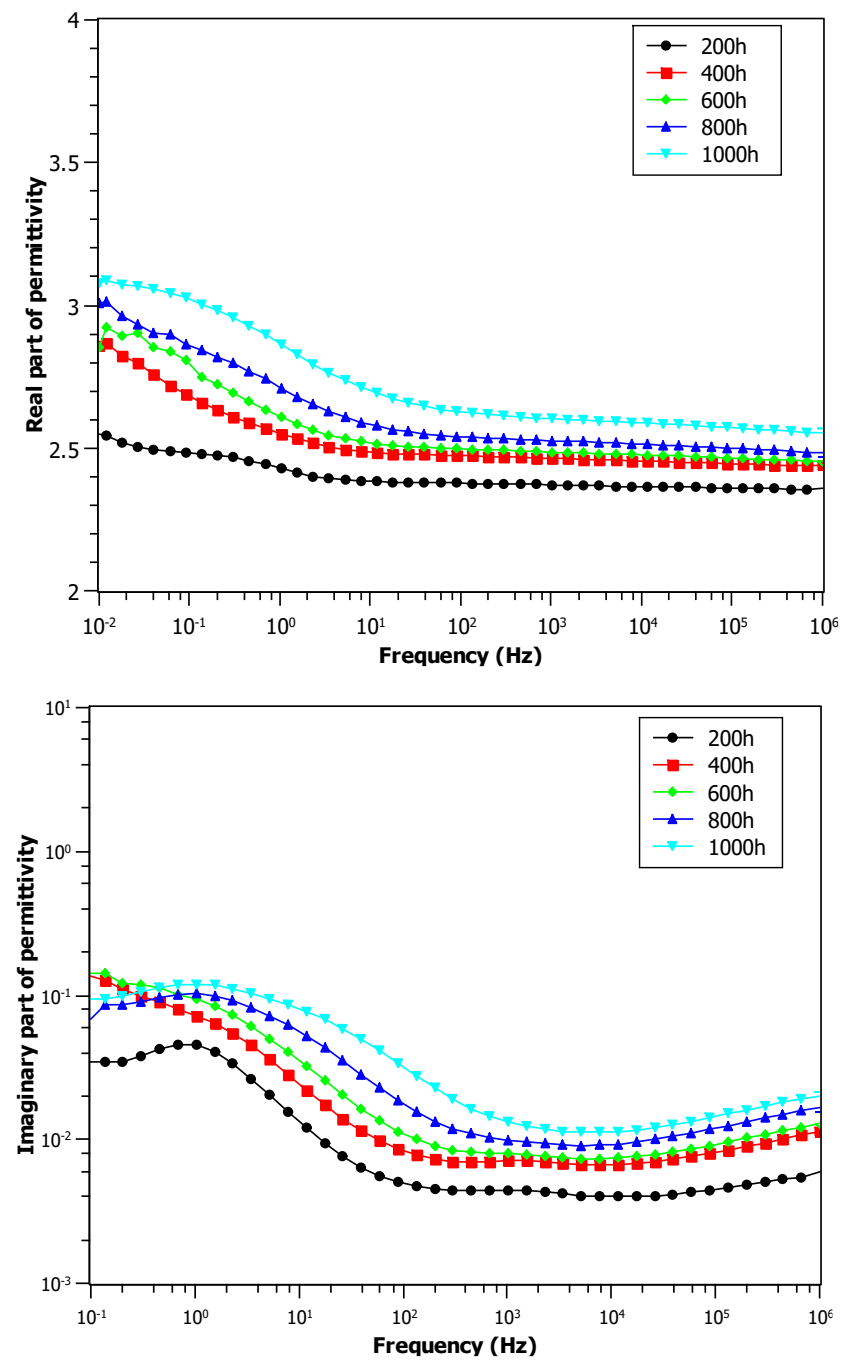

Fig. 2. Dielectric spectra as a fuction of frequency of real (a) and imaginary (b) part of permittivity. Dose rate $1.06 \mathrm{kGy} / \mathrm{h}$ and temperature $85^{\circ} \mathrm{C}$. Tests made at delivery time

In both the aging conditions here analyzed one can observe a dramatic change in dielectric response imputed to this physical phenomenon.

Referring to the real part of permittivity after storage (Figs. 3.a and 4.a), one can observe a very low raise of $\varepsilon$ ' with aging, particularly in the high frequency range. On the contrary, in the lowest frequency range, variation of $\varepsilon$ ' is wider due to strong electrode polarization (EP). Also in this case, EP is dependent on the dose rate imposed during the aging process: the higher the irradiation dose the higher the EP.

The imaginary part of permittivity spectra (Figs. 3.b and 4.b) show very similar behavior in terms of trend and magnitude except for the interfacial polarization area.
In fact, in the high frequency range $\varepsilon$ " value is almost the same between the two spectra (see Figs. 3.b and 4.b), suggesting that the radiation aging process only affect the short-term dielectric loss factor of the insulation.

This can be explained considering $[6,7]$ that irradiation causes the formation of radicals in both the crystalline and amorphous part of the polymer. In fact, radicals in the amorphous part are able to bond each other or with other reactive molecules (e.g. oxygen). ,On the contrary, radicals in the crystalline phase remain trapped inside the crystal and take some time to migrate towards the crystalline/amorphous interface where they are able to bond and react with other reactive species.

This behavior is the theoretical basis of the post-irradiation effect which can bring to degradation phenomena (e.g. oxidation, molecular rearrangement, ...) even when the irradiation source is turned off.

Higher dose rates can cause higher radical formation with respect to lower dose rate aging in both the phases of the polymeric blend. In the short-term analysis (Figs. 1 and 2), radicals made by the high dose rate (Fig. 2) in the amorphous phase can bond with the atmospheric oxygen which is not enough to react with the all reactive species formed by radiation, inducing the already discusses DLO effect.

On the other hand, radicals formed in the crystalline phase remain trapped and after some time they lead to various degradation phenomena, even when the radiation source is off. This occurs until the polymer does reach the maximum of its degradation, above which it cannot exceed due to the loss of reactive species.

In the same way, lower radiation aging can cause the formation of radicals in both phases and the saturation of the degradability of the polymer here considered. This can be considered as the reason why the dielectric behavior of differently aged cables can appear the same between the two aging conditions.

The only difference can be evaluated in the different frequency of the peak referred to the interfacial polarization, which can be likely imputed, as stated above, to the changed molecular arrangement caused by the different aging treatment.

\section{CONCLUSIONS}

This research investigates the post-irradiation effect of XLPEinsulated low voltage cables used in nuclear power plants.

Aging has been performed at the same temperature and under two different dose rates in order to evaluate the behavior of the insulation blend under different radiation doses.

Tests have been performed at delivery time and after five years of storage during which radiation source has been turned off in order to evaluate the possible post-irradiation effect inside the insulation.

The main outcome of this research is that the electrical response of irradiated cables can be remarkably different under different dose rates in the short-term analysis. This difference is almost negligible after some time during which the irradiation source is turned off due to post irradiation effects which occur inside the insulation.

The different radiation dose rate only arises in the different molecular arrangement but does not cause any difference in 
terms of dielectric loss order of magnitude and high-frequency trend. This behavior has been imputed to the polymer degradation which can saturate after some time, so that radicals made by higher dose rate does not affect long-term postirradiation electrical behavior of cables.

\section{ACKNOWLEDGMENT}

The authors are grateful to Simona Addamo and Andrea Tilli for the help in carrying out permittivity measurements.

\section{REFERENCES}

[1] International Atomic Energy Agency, "Assessing and managing cable aging in nuclear power plants", 2012.

[2] E. Linde, L. Verardi, D. Fabiani, U.W. Gedde, "Dielectric spectroscopy as a condition monitoring technique for cable insulation based on crosslinked polyethylene", Polymer Testing.
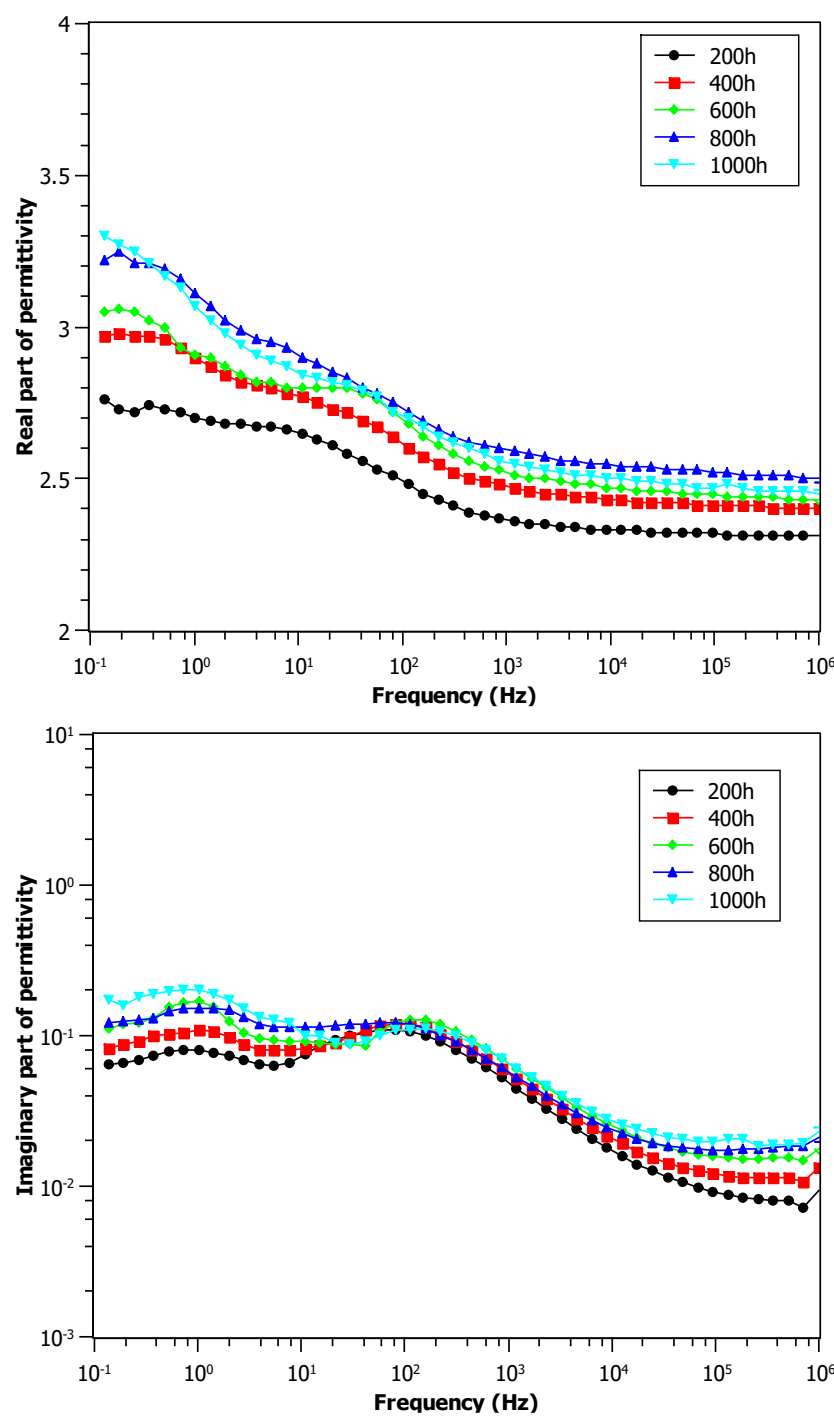

Fig. 3. Dielectric spectra as a fuction of frequency of real (a) and imaginary (b) part of permittivity. Dose rate $0.42 \mathrm{kGy} / \mathrm{h}$ and temperature $85{ }^{\circ} \mathrm{C}$. Tests made after five years of storage.
[3] J. Wise, K.T. Gillen, R.L. Clough, Quantitative model for the time development of diffusion-limited oxidation profiles, Polymer, Volume 38, Issue 8, 1997, Pages 1929-1944.

[4] D. Fabiani, S.V. Suraci, S. Bulzaga, "Aging investigation of low-voltage cable insulation used in nuclear power plants", EIC 2018, San Antonio, Texas.

[5] E. Suljovrujic, "Post-irradiation effects in polyethylenes irradiated under various atmospheres", Radiation Physics and Chemistry.

[6] P. Ishai, M. Talary, A. Caduff, E. Levy, Y. Feldman, "Electrode polarization in dielectric measurements: a review", Measurement science and technology, 2013.

[7] S. Nakamura, F. Murabayashi, K. Iida, G. Sawa, and M. Ieda, "Degradation of dielectric properties of polyethylene by combined irradiation and thermal stresses", IEEE Transactions on Electrical Insulation, vol. 22, no. 6, pp. 715-720, 1987
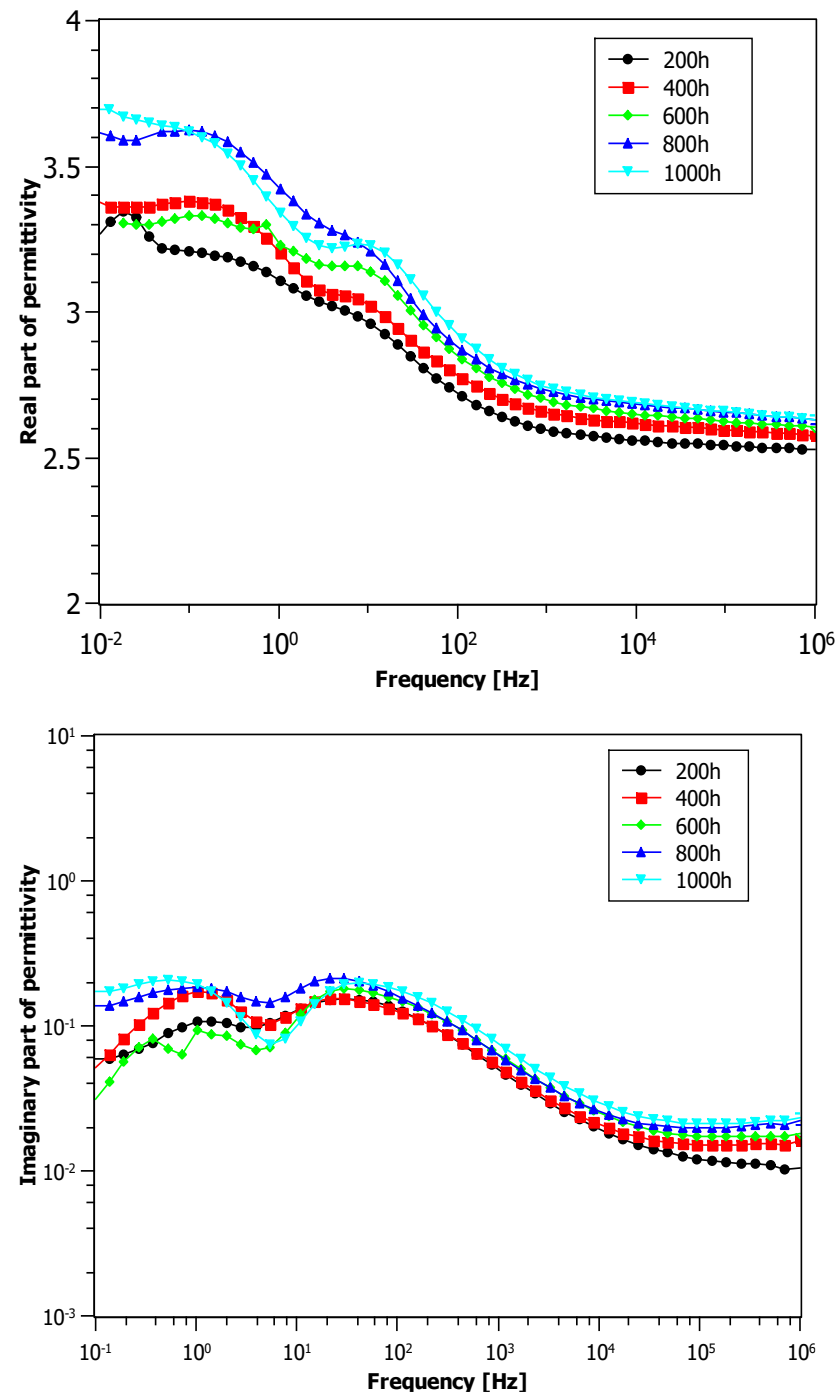

Fig. 4. Dielectric spectra as a fuction of frequency of real (a) and imaginary (b) part of permittivity. Dose rate $1.06 \mathrm{kGy} / \mathrm{h}$ and temperature $85{ }^{\circ} \mathrm{C}$. Tests made after five years of storage. 